\title{
Terahertz Spectroscopy and Brewster Angle Reflection Imaging of Acoustic Tiles
}

\author{
Patrick Kilcullen, ${ }^{1}$ Mark Shegelski, ${ }^{1}$ MengXing $\mathrm{Na}^{2}$ David Purschke, ${ }^{2}$ Frank Hegmann, ${ }^{2}$ and \\ Matthew Reid ${ }^{1}$ \\ ${ }^{1}$ Department of Physics, University of Northern British Columbia, Prince George, BC, Canada V2N 4Z9 \\ ${ }^{2}$ Department of Physics, University of Alberta, Edmonton, AB, Canada T6G 2E1 \\ Correspondence should be addressed to Patrick Kilcullen; kilcull@unbc.ca
}

Received 10 November 2016; Accepted 5 January 2017; Published 8 March 2017

Academic Editor: Arnaud Cuisset

Copyright (c) 2017 Patrick Kilcullen et al. This is an open access article distributed under the Creative Commons Attribution License, which permits unrestricted use, distribution, and reproduction in any medium, provided the original work is properly cited.

\begin{abstract}
A Brewster angle reflection imaging apparatus is demonstrated which is capable of detecting hidden water-filled voids in a rubber tile sample. This imaging application simulates a real-world hull inspection problem for Royal Canadian Navy Victoria-class submarines. The tile samples represent a challenging imaging application due to their large refractive index and absorption coefficient. With a rubber transmission window at approximately $80 \mathrm{GHz}$, terahertz (THz) sensing methods have shown promise for probing these structures in the laboratory. Operating at Brewster's angle allows for the typically strong front surface reflection to be minimized while also conveniently making the method insensitive to air-filled voids. Using a broadband $\mathrm{THz}$ time-domain waveform imaging system (THz-TDS), we demonstrate satisfactory imaging and detection of water-filled voids without complicated signal processing. Optical properties of the tile samples at low $\mathrm{THz}$ frequencies are also reported.
\end{abstract}

\section{Introduction}

The transparency of many dry, nonconductive materials to radiation at $\mathrm{THz}$ frequencies has allowed new applications of broadband $\mathrm{THz}$ pulses to nondestructive evaluation (NDE) and the imaging of concealed objects and surfaces [1-3]. THz radiation is electromagnetic radiation found in roughly the $0.1 \mathrm{THz}$ to $10 \mathrm{THz}$ range, between the microwave and infrared portions of the electromagnetic spectrum. $\mathrm{THz}$ radiation is nonionizing and has submillimeter wavelength, making it a competitive imaging technology to X-rays without having the associated health risks [4]. For example, it has proven suitable for imaging defects in foam insulation within the fuel tanks of the NASA space shuttle [5]. THz reflection tomography [6] has been demonstrated using coherently detected $\mathrm{THz}$ pulses and has been used for applications ranging from the imaging of delaminations [7] and under paint corrosion $[8,9]$ to art conservation [10]. $\mathrm{THz}$ radiation is also extremely sensitive to water which absorbs it strongly in the far infrared. This has been exploited to image water content and diffusion in cork [11] and to identify concealed water-filled voids [7].

The motivation for this project was to investigate the feasibility of a reflection imaging system for the hull inspection of Royal Canadian Navy Victoria-class submarines (Figure 1). In order to operate with enhanced stealth, these vessels are fitted with an acoustic rubber tiling for the purposes of absorbing emanating noise and providing sonar cloaking. The design of the acoustic tiles generally features internal voids for enhanced acoustic absorption. These tiles, as well as a connective grouting compound, thus form an opaque covering over virtually the entire hull surface.

In evaluating the integrity of the pressurized hull, it is of paramount importance to discern states of corrosion of the underlying steel in addition to delamination and seawater ingress within the tile voids and grouting interfaces. A nondestructive means of on-site inspection with $\mathrm{THz}$ radiation has therefore been sought after. Such nondestructive 


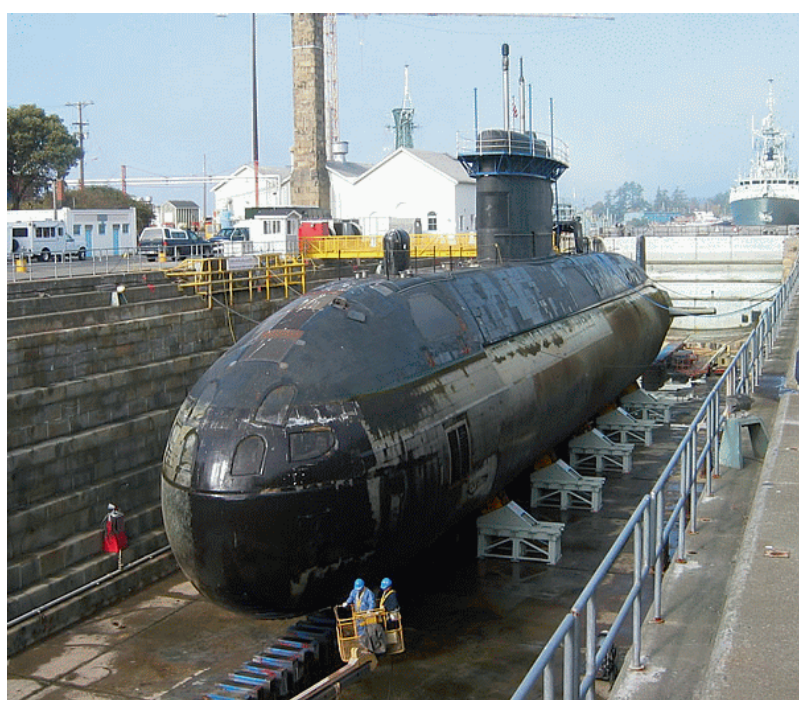

Figure 1: The HMCS Victoria, one of Canada's four Victoria-class submarines, shown in dry dock (Department of National Defence photo).

evaluation would be done in practice from the outside surface of the hull, with the radiation reflecting off the metal subsurface or intermediate tile layers.

It has been observed that, as a prelude to further corrosion, sites of water ingress are typically characterized by the internal tile voids becoming flooded with seawater. The ability to detect hidden water-filled voids could therefore constitute an early warning for corrosion, and an easy signal for tile replacement and regrouting.

Due to security reasons that precluded the use of actual tile samples, representative rubber tile samples or "analogue tiles" were used in the laboratory for this study. Each of the tile samples was approximately 4 inches square by 1 inch thick and consisted of three laminated layers (see Figure 5, oval inset). Engineered voids were simulated by holes drilled into the middle layer.

\section{THz Apparatus}

The THz-TDS system used in this work was a high speed Picometrix T-Ray 4000 system, generating linearly polarized, pulsed $\mathrm{THz}$ radiation detected in an 80 ps window, with a bandwidth of approximately $2 \mathrm{THz}$, modified to operate at a 1000 waveforms/sec acquisition rate. The emitter and detector are fibre-coupled photoconductive switches, allowing the system to be easily mounted in a raster-scanning configuration for the imaging studies.

Example $\mathrm{THz}$ signals from the $\mathrm{THz}$-TDS system are presented in Figure 2 for transmission through both air and analogue tile. The time-domain waveforms presented in Figure 2(a) were stitched from several waveform observations (80 ps duration) obtained from the device. After transmission through the tile sample, the initial $\mathrm{THz}$ pulse is delayed and strongly attenuated. A window of $\mathrm{THz}$ transmission for the analogue rubber can be seen in Figure 2(b) centered at approximately $80 \mathrm{GHz}$.

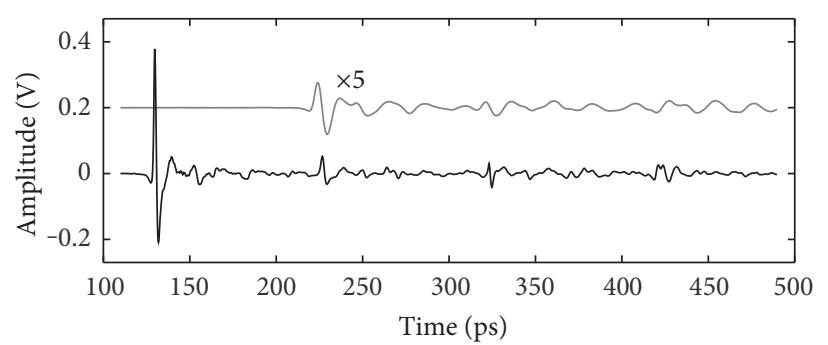

(a)

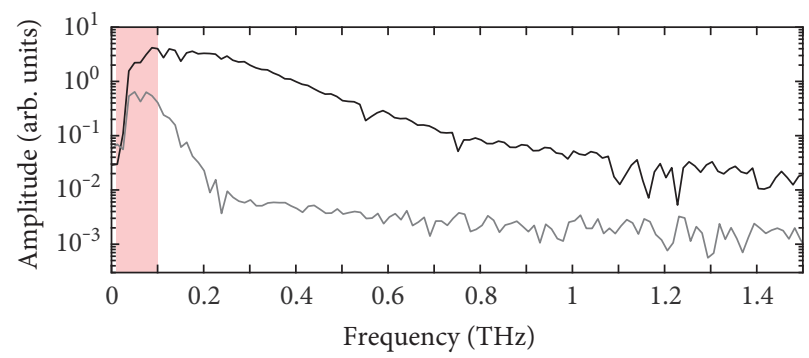

(b)

Figure 2: Example $\mathrm{THz}$ signals produced by the THz-TDS apparatus. (a) Time-domain comparison of a thru-air $\mathrm{THz}$ waveform (lower, black) and a thru-tile waveform (upper, gray). The thru-tile signal has been offset and magnified $\times 5$ for clarity. (b) Log scale frequency domain comparisons corresponding to the thru-air (black) and thru-tile (gray) waveforms. The 0.01 to $0.10 \mathrm{THz}$ frequency band used for the transmission image has been highlighted in red.

\section{Imaging Experiments}

The large refractive index and absorption of the analogue tile samples was extracted from transmission and reference time-domain measurements using the method presented in $[12,13]$. The extracted absorption coefficient and refractive index are shown in Figure 3.

Transmission imaging scans were initially taken of the analogue sample tiles. A direct transmission image taken of the internal air-filled void structure of tile sample "A1-8" is shown in Figure 4 which was generated from waveform data captured at each position. The color map corresponds to the amplitude of the transmitted spectral content in the $0.01 \mathrm{THz}$ to $0.10 \mathrm{THz}$ range (highlighted in Figure 2(b)) computed from the Fourier transform of the time-domain data. Variation in transmission is primarily attributed to a nonuniform bonding glue application between layers. The two internal voids are clearly observable from transmission minima at their edges due to the deflection and subsequent trapping of the penetrating radiation.

The large refractive index of the analogue tiles leads to a large front surface reflection at normal incidence through air at $\mathrm{THz}$ frequencies. In addition for reflection imaging, the large absorption of the material diminishes the amplitude of collected signals which must pass through any absorptive layers twice before detection. For example, at $80 \mathrm{GHz}$ and at normal incidence, the expected reflected intensity coefficient from the top surface of a water-filled void within our sample would be 0.0146 , 


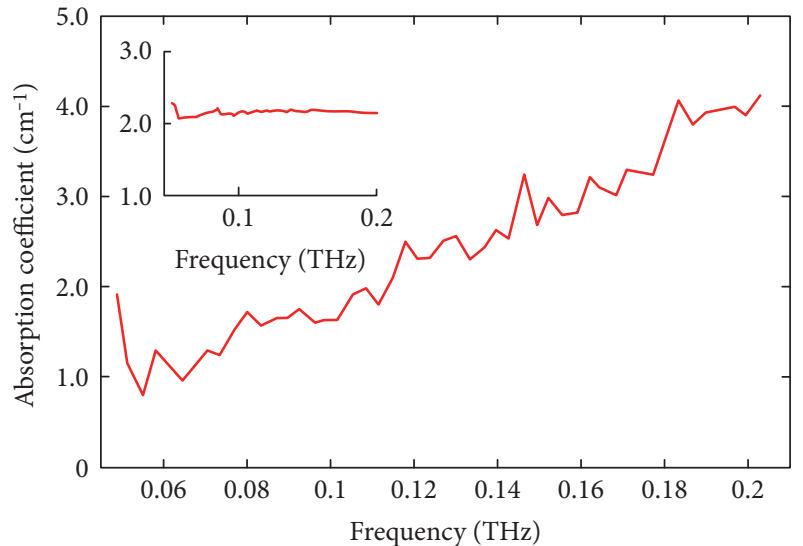

FIGURE 3: Propagation properties of the analogue tiles. The absorption coefficient is plotted as a function of frequency, shown in units of inverse centimeters. Inset: index of refraction, plotted as a function of frequency. These measurements were computed using the method presented in $[12,13]$.

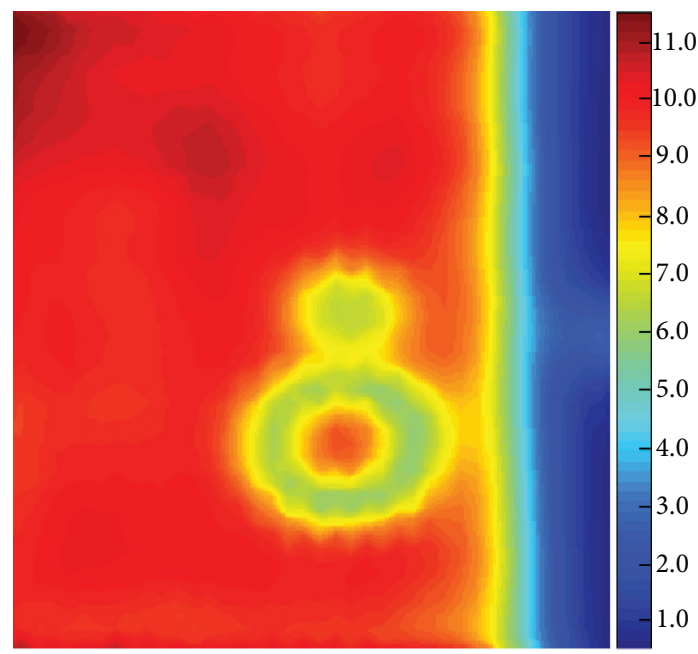

FIgURE 4: Spectral content transmission image $(0.01 \mathrm{THz}$ to $0.10 \mathrm{THz}$ ) of tile sample "A1-8" with air-filled voids taken at a grid resolution of $2 \mathrm{~mm}$. Transmitted energy is shown colored from blue (low transmission) to red (high transmission) with the scale shown in arbitrary units. Both circular voids are discernible. The large and small cylindrical voids of this sample are $2.2 \mathrm{~cm}$ and $0.75 \mathrm{~cm}$ in diameter, respectively, with a separation of $0.8 \mathrm{~cm}$. The transmission cut-off on the right hand side of the image was caused by metal apparatus used to hold the tile sample.

whereas the expected reflected intensity from the front surface would be greater by a factor of 9.5. A value of $n_{\text {water }}=3.98+i 2.13$ at $80 \mathrm{GHz}$ [14] was used to estimate these values, along with the data of Figure 3 .

Due to the large refractive index and strong absorption of the analogue tiles, we attempted reflection imaging at $80 \mathrm{GHz}$ from the top rubber-rubber tile interface, located at a depth of approximately 0.25 inches below the top tile surface (see Figure 5, oval inset). Operating at the Brewster angle with TM-polarization minimized the front surface reflection and maximized signal penetration.

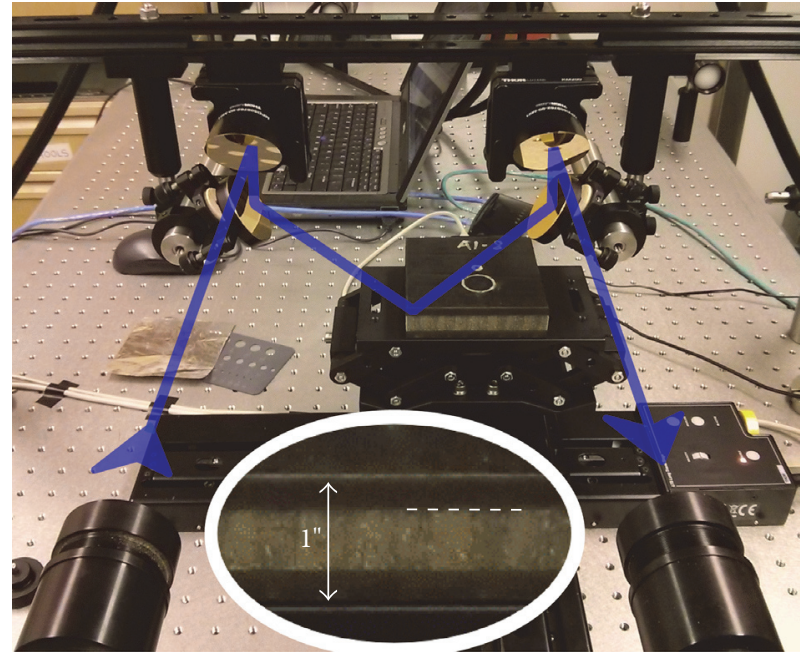

FIgURE 5: Sample tile "A1-8" positioned ready for scanning. The two black cylinders on the left and right are the Teflon-collimating lens enclosures of the $\mathrm{THz}$ emitter and detector, respectively. The path of the $\mathrm{THz}$ beam is highlighted in blue. Upon exiting the transmitter the $\mathrm{THz}$ radiation is linearly polarized horizontally across the optical table. The rectangular tile sample is positioned on top of a precision lab jack and two $X-Y$ positioning stages. Oval inset: an edge closeup of the three-layered tile sample. The dashed horizontal line indicates the target depth of the reflection imaging at the first rubber-rubber interface.

Brewster angle microscopy and imaging was developed to exploit dielectric contrast to probe surface interfaces [15], where it has been exceedingly successful at investigating even monolayers at surface interfaces $[15,16]$. We employ the Brewster angle here to make our imaging set-up as insensitive as possible to the surface interface, while exploiting dielectric contrast to probe subsurface interfaces, which would otherwise be obscured by strong front-surface back reflections.

Our Brewster's angle imaging apparatus is photographed in Figure 5. Off-axis parabolic mirrors (F/3) were used to focus the incident radiation into a narrow beam incident at the $80 \mathrm{GHz}$ Brewster's angle of $63^{\circ}$. The analogue tile samples were controlled in both horizontal directions using a pair of motorized translation stages, while the emitter and detector positions remained fixed.

Raster images of the analogue tile samples were produced by capturing individual waveforms as the sample was repeatedly repositioned at the focus. Using 1000 averages for each collected waveform, our system produced imaging scans at a rate of approximately 7 seconds per pixel. The coherent detection allowed for temporal alignment of the acquired waveforms in order for a particular depth of reflection to be selected. Temporal alignment was achieved from an estimation of the optical path length of a single-pass reflection of the beam at the first interface at Brewster's angle. Raster waveform data was then used to produce gray scale sample images by first truncating the waveforms to a $25 \mathrm{ps}$ window containing the peak of the reflected pulse, and subsequently extracting the integrated spectral 


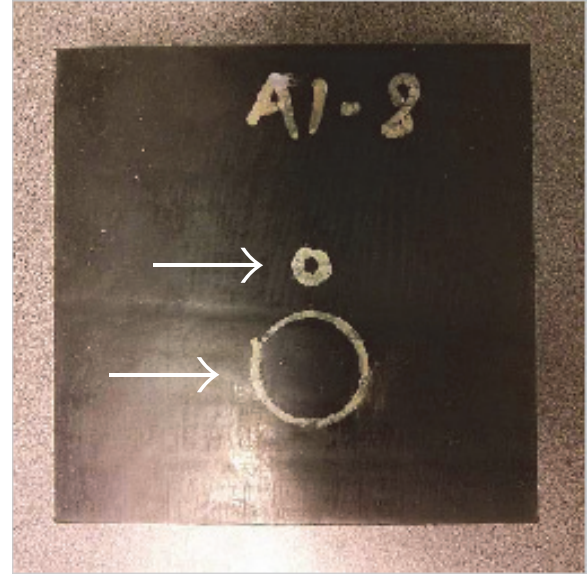

(a)

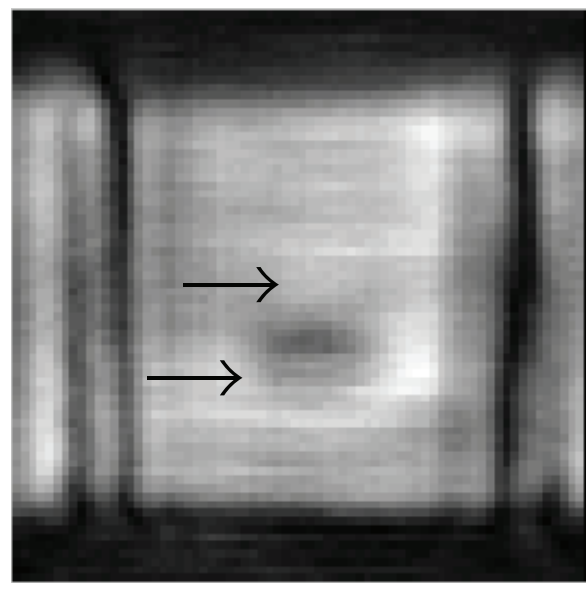

(c)

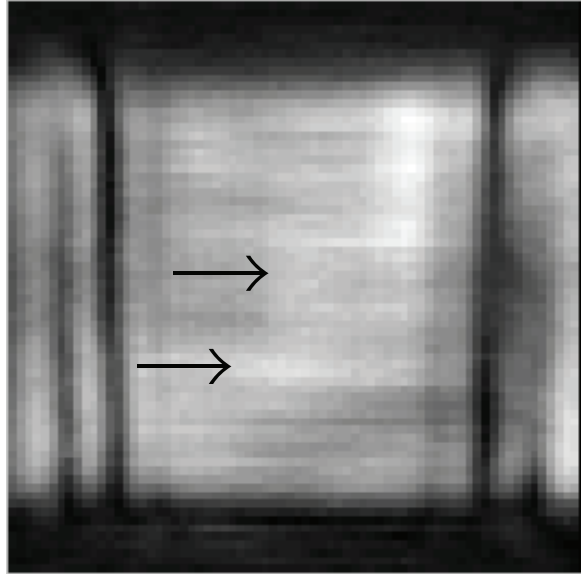

(b)

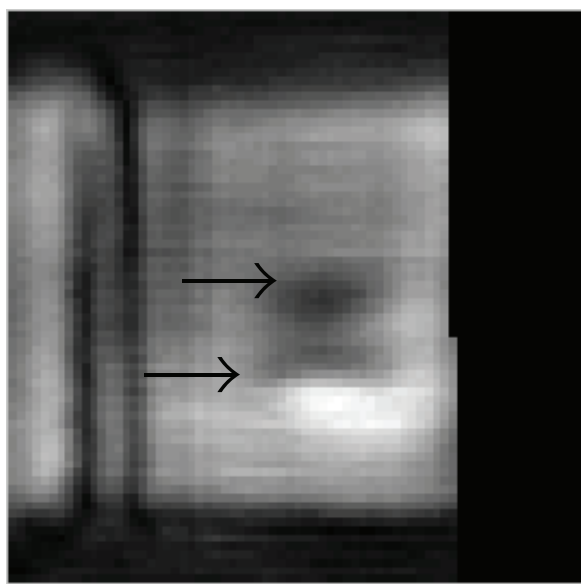

(d)

Figure 6: Spectral content reflection images $(70 \times 70$ pixels $)$ of tile sample "A1- 8 " in the range of 80 GHz. Positions of the voids are highlighted by the arrows. (a) Color photograph. (b) THz reflection image. (c) THz reflection image with larger hole fully injected with water. (d) THz reflection image with both holes fully injected. Unfortunately, a computer fault interrupted scan (d) before completion. The vertical dark bands at the sides of the tiles are edge effects due to the oblique incidence angle of the THz beam, which also distorts the shapes of the hidden defects horizontally. The acquisition time for each image was approximately 10 hours, limited primarily by the electronics of the raster scanning.

content of the signal in the vicinity of $80 \mathrm{GHz}$ through the use of a discrete Fourier transform.

Brewster angle reflection images of the sample tile "A1-8" are shown in Figure 6. Our image progression simulates the imaging effect of water ingress into the internal voids. To achieve this, a syringe was used to inject tap water into the voids until they were completely flooded. The progression in Figure 6 clearly shows the sensitivity of our technique to the detection of flooded internal voids in the sample tiles. The oblong appearance of the flooded circular voids is due to the oblique angle of incidence of the $\mathrm{THz}$ beam in the Brewster's angle imaging configuration.

A particular advantage of imaging at the Brewster angle for this application is that those internal voids not contaminated by fluid ingress are invisible. This may be understood by considering the Brewster angle path of the $\mathrm{THz}$ beam illustrated in Figure 7 for the cases of rubber-rubber, rubber-air, and rubber-water interfaces. In particular, a beam which penetrates the top rubber layer and enters a void must exit the cavity ceiling at the angle of incidence (Figure 7(b)), which therefore implies total transmission by the time reversal of the Brewster property. Due to the low dielectric contrast between the tile layers, such near-optimal transmission is also observed at the contiguous rubber-rubber interfaces of the sample (Figure $7(\mathrm{a})$ ). Reflected signals from the first interface therefore do not exhibit contrast between the uncontaminated voids and the contiguous analogue rubber media surrounding them. Upon fluid ingress into the voids, the total transmission property is no longer valid at the cavity interface, and the result is an increased reflected signal from the interface (Figure 7(c)).

We hypothesize that the mostly "dark" appearance of the water-filled voids is due to the destructive Bragg interference between the reflected flooded cavity interface and the front surface reflection, shown by the two dashed rays in Figure $7(\mathrm{c})$.

Front surface reflections are still encountered even when operating at Brewster's angle in practice and are illustrated by 


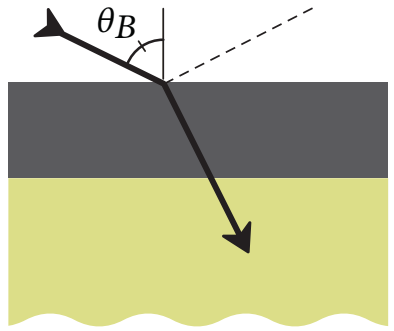

(a)

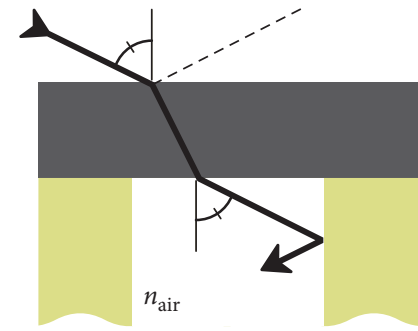

(b)

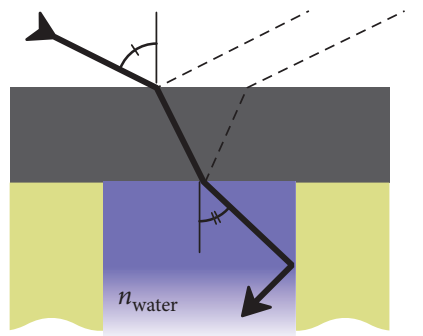

(c)

Figure 7: Ray diagram illustration of the Brewster's angle reflection geometry for (a) a continuous rubber interface, (b) an air-filled void, and (c) a water-filled void. The dashed lines indicate significant reflected rays.

the topmost dashed rays in Figures $7(\mathrm{a}), 7(\mathrm{~b})$, and $7(\mathrm{c})$. Despite the temporal alignment and truncation of our acquired waveforms, the reflected pulse windows still contained atmospheric "ringing" from the front surface reflected pulse. Interpreting this residual front surface signal as a noise floor for our apparatus, we obtain a signal to noise ratio (SNR) of 1.64 compared to the peak to peak amplitude of the reflected pulse from the void interface. This value is a lower bound for the SNR, as the front surface back reflection does not change significantly over the surface of the samples.

\section{Conclusion}

We were able to observe hidden water-filled voids through reflection mode imaging without complicated image processing. Successful water-filled void detection was possible at a depth of 0.25 inches beneath a layer of highly absorbing rubber in the laminated analogue tile sample. By operating at the Brewster angle, front surface reflections could be minimized with the added advantage of making uncontaminated voids invisible upon scanning.

A low dielectric contrast between the top and middle layers of the analogue rubber sample allowed for identical transmission between the contiguous rubber and cavity spaces at the depth of the first interface. We note that, for cases in which the dielectric contrast between the top and middle layer is significant, such identical transmission could theoretically be achieved via tuning of the reflection imaging angle through the Fresnel equations.

\section{Conflicts of Interest}

The authors declare that they have no conflicts of interest.

\section{Acknowledgments}

This research was carried out in the course of a larger collaboration between the authors' research group at UNBC, the University of Alberta, McGill University, and Defence Research and Development Canada (DRDC). In addition to the authors, the members of this collaboration included Dr. David Cooke, David Valverde, and Lauren Gingras at McGill University and Rod McGregor at DRDC. The authors wish to acknowledge funding for this project from DRDC, NSERC, and the AITF Strategic Chair Program.

\section{References}

[1] K. H. Jin, Y.-G. Kim, S. H. Cho, J. C. Ye, and D.-S. Yee, "High-speed terahertz reflection three-dimensional imaging for nondestructive evaluation," Optics Express, vol. 20, no. 23, pp. 25432-25440, 2012.

[2] T. D. Dorney, W. W. Symes, R. G. Baraniuk, and D. M. Mittleman, "Terahertz multistatic reflection imaging," Journal of the Optical Society of America. A, vol. 19, no. 7, pp. 1432-1442, 2002.

[3] P. U. Jepsen, D. G. Cooke, and M. Koch, "Terahertz spectroscopy and imaging-Modern techniques and applications," Laser \& Photonics Reviews, vol. 5, no. 1, pp. 124-166, 2011.

[4] D. Saeedkia, Handbook of terahertz technology for imaging, sensing and communications, WP Woodhead Publishing, Oxford, England Philadelphia, Pennsylvania, 2013.

[5] D. Zimdars, J. S. White, G. Stuk, A. Chernovsky, G. Fichter, and S. Williamson, "Large area terahertz imaging and nondestructive evaluation applications," Insight, vol. 48, no. 9, pp. 537-537, 2006.

[6] D. Mittleman, R. Jacobsen, and M. Nuss, "T-ray imaging," IEEE Journal of Selected Topics in Quantum Electronics, vol. 2, no. 3, pp. 679-692, 1996.

[7] D. Zimdars, J. White, G. Sucha et al., "Terahertz for military and security applications V," in Conference on Terahertz for Military and Security Applications V, vol. 6549, p. 54906, 2007.

[8] R. Anastasi and E. Madaras, "Terahertz NDE for under paint corrosion detection and evaluation," in AIP Conference Proceedings, vol. 820, pp. 515-522, 2006.

[9] R. F. Anastasi, E. I. Madams, J. P. Seebo et al., "Terahertz NDE application for corrosion detection and evaluation under shuttle tiles," in Proc. SPIE, vol. 6531, p. W5310, 2007.

[10] J. B. Jackson, J. Bowen, G. Walker et al., "A Survey of Terahertz Applications in Cultural Heritage Conservation Science," IEEE Transactions on Terahertz Science and Technology, vol. 1, no. 1, pp. 220-231, 2011.

[11] Y. L. Hor, J. F. Federici, and R. L. Wample, "Nondestructive evaluation of cork enclosures using terahertz/millimeter wave spectroscopy and imaging," Applied Optics, vol. 47, no. 1, pp. 72-78, 2008.

[12] L. Duvillaret, F. Garet, and J.-L. Coutaz, "A reliable method for extraction of material parameters in terahertz time-domain spectroscopy," IEEE Journal of Selected Topics in Quantum Electronics, vol. 2, no. 3, pp. 739-746, 1996.

[13] I. Pupeza, R. Wilk, and M. Koch, "Highly accurate optical material parameter determination with $\mathrm{THz}$ time-domain spectroscopy," Optics Express, vol. 15, no. 7, pp. 4335-4350, 2007. 
[14] J. T. Kindt and C. A. Schmuttenmaer, "Far-infrared dielectric properties of polar liquids probed by femtosecond terahertz pulse spectroscopy," The Journal of Physical Chemistry, vol. 100, no. 24, pp. 10373-10379, 1996.

[15] D. Hoenig and D. Moebius, "Direct visualization of monolayers at the air-water interface by Brewster angle microscopy," The Journal of Physical Chemistry, vol. 95, no. 12, pp. 45904592, 1991.

[16] M. Harke, R. Teppner, O. Schulz, H. Motschmann, and H. Orendi, "Description of a single modular optical setup for ellipsometry, surface plasmons, waveguide modes, and their corresponding imaging techniques including Brewster angle microscopy," Review of Scientific Instruments, vol. 68, no. 8, pp. 3130-3134, 1997. 

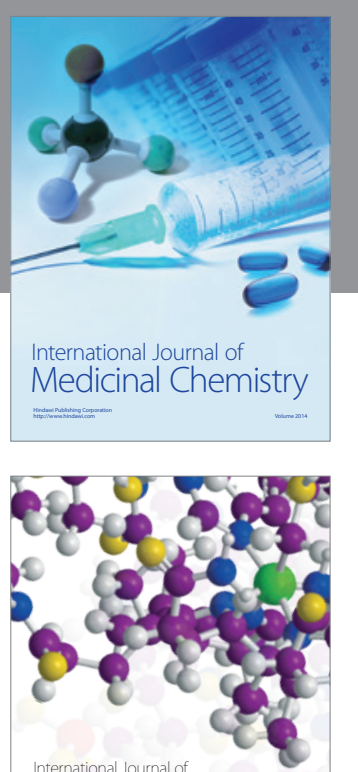

Carbohydrate Chemistry

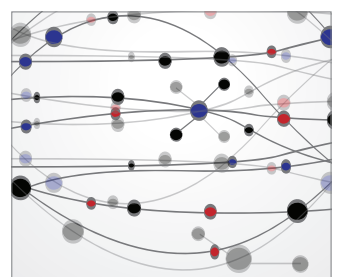

The Scientific World Journal
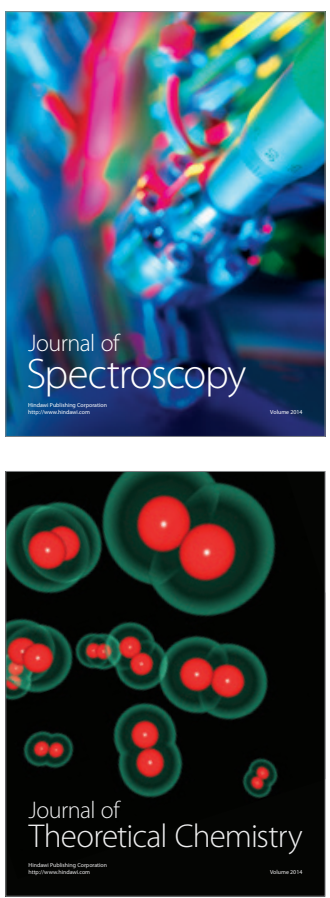
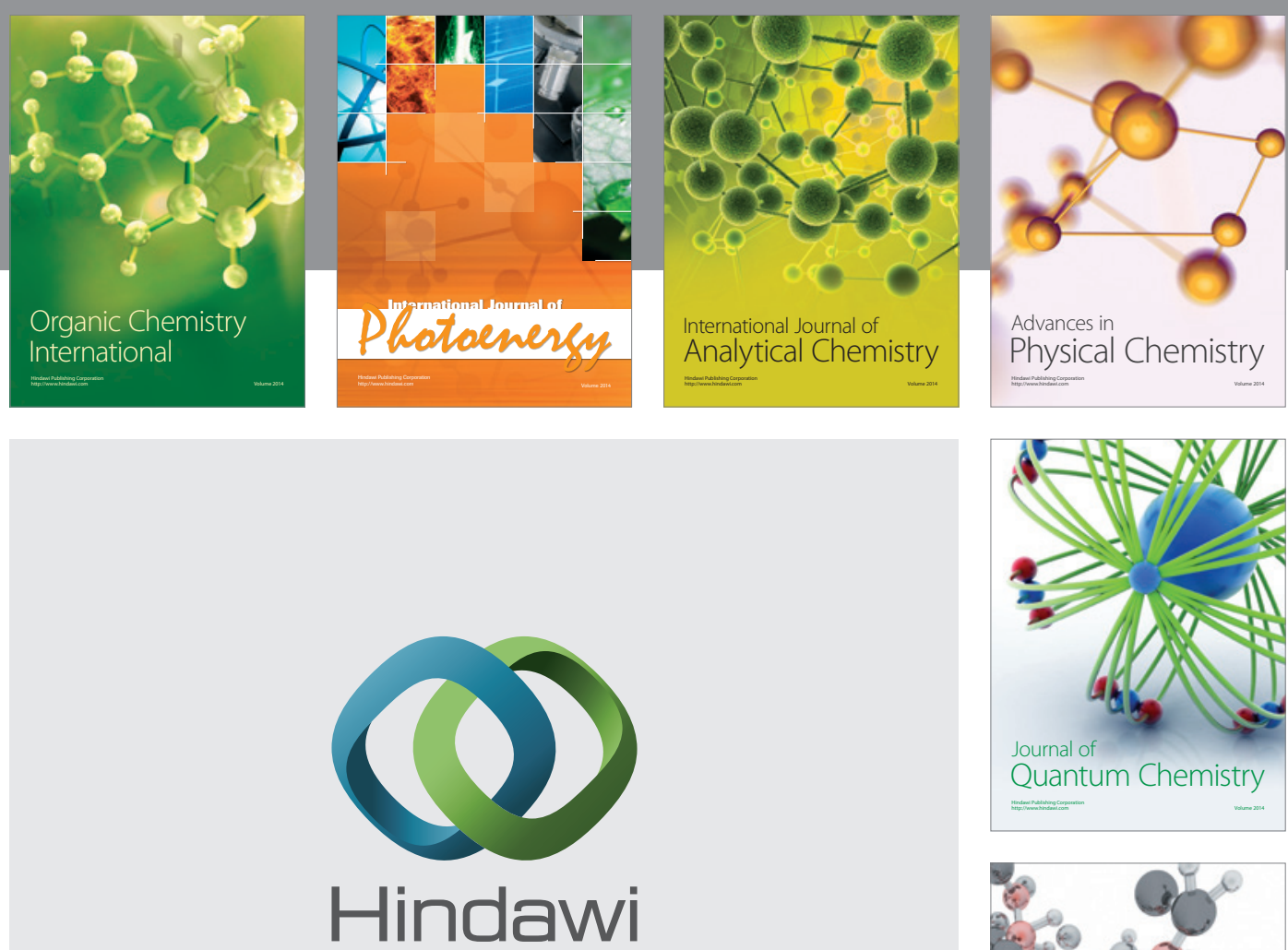

Submit your manuscripts at

https://www.hindawi.com

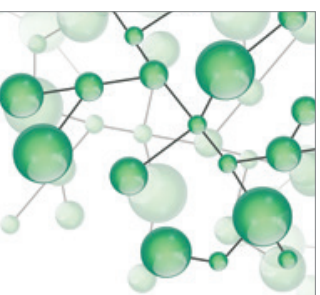

International Journal of

Inorganic Chemistry
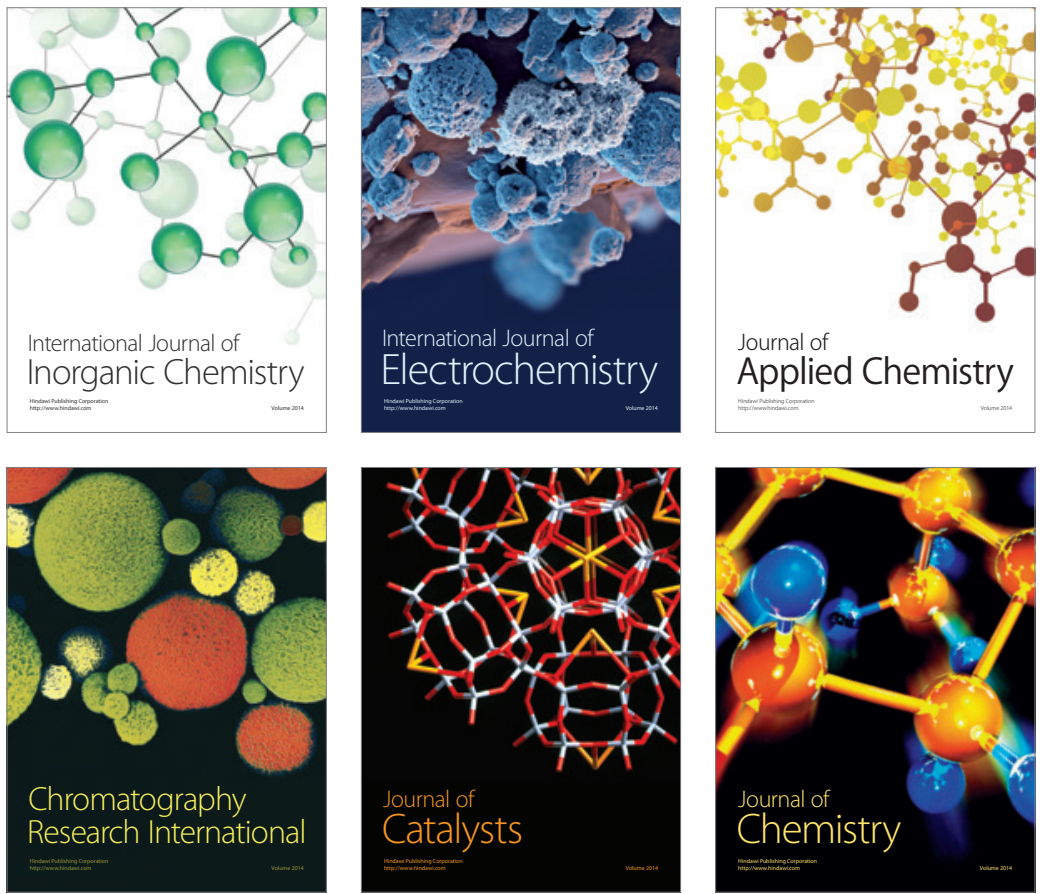

Journal of

Applied Chemistry
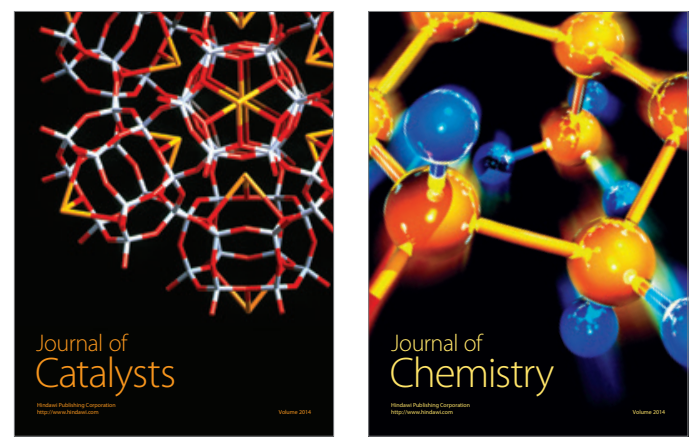
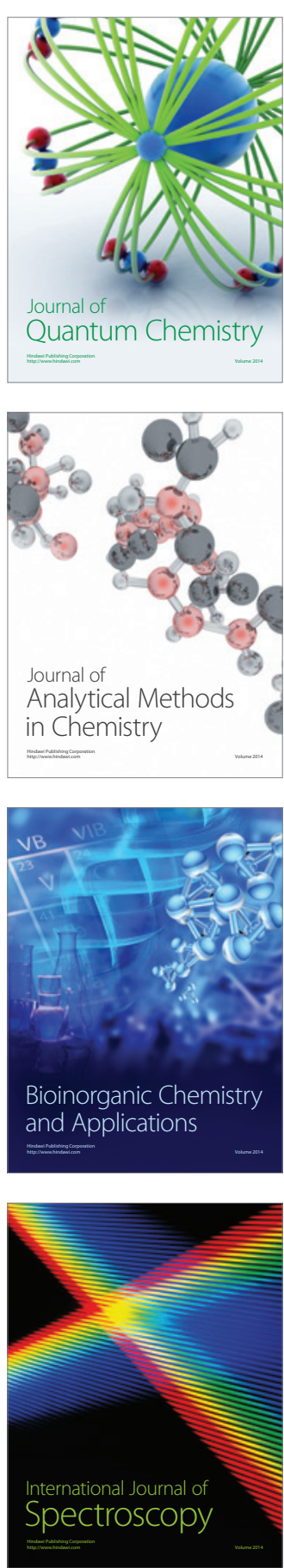